\title{
Research on the Relationships between Endogenous Biomarkers and Exogenous Toxic Substances of Acute Toxicity in Radix Aconiti
}

\author{
Haonan Zhou, Pengjie Zhang, Zhiguo Hou, Jiabin Xie, Yuming Wang, Bin Yang, Yanyan Xu * \\ and Yubo Li * \\ Tianjin State Key Laboratory of Modern Chinese Medicine, School of Traditional Chinese Materia Medica, \\ Tianjin University of Traditional Chinese Medicine, 312 Anshan West Road, Tianjin 300193, China; \\ zhnwork09@163.com (H.Z.); 13752586596@163.com (P.Z.); hzgssh@163.com (Z.H.); 18600893359@163.com (J.X.); \\ wangyuming226@163.com (Y.W.); yang3023008@163.com (B.Y.) \\ * Correspondence: xyytjutcm@163.com (Y.X.); yaowufenxi001@sina.com (Y.L.); Tel./Fax: +86-22-59596221 (Y.L.) \\ Academic Editor: Marcello Iriti \\ Received: 13 October 2016; Accepted: 21 November 2016; Published: 25 November 2016
}

\begin{abstract}
Radix Aconiti, a classic traditional Chinese medicine (TCM), has been widely used throughout China for disease treatment due to its various pharmacological activities, such as anti-inflammatory, cardiotonic, and analgesic effects. However, improper use of Radix Aconiti often generated severe acute toxicity. Currently, research on the toxic substances of Radix Aconiti is not rare. In our previous study, acute toxic biomarkers of Radix Aconiti have been found. However, few studies were available to find the relationships between these endogenous biomarkers and exogenous toxic substances. Therefore, in this study, toxic substances of Radix Aconiti have been found using UPLC-Q-TOF-MS technology. Then, we used biochemical indicators as a bridge to find the relationships between biomarkers and toxic substances of Radix Aconiti through Pearson correlation analysis and canonical correlation analysis (CCA). Finally, the CCA results showed that LysoPC(22:5) is related to 14-acetyl-talatisamine, mesaconitine, talatisamine and deoxyaconitine in varying degrees; L-acetylcarnitine is negatively correlated with deoxyaconitine and demethyl-14-acetylkaracoline; shikimic acid has a good correlation with karacoline, demethyl-14-acetylkaracoline and deoxyaconitine; and valine is correlated with talatisamine and deoxyaconitine. Research on these relationships provides an innovative way to interpret the toxic mechanism of traditional Chinese medicine, and plays a positive role in the overall study of TCM toxicity.
\end{abstract}

Keywords: Radix Aconiti; toxic substances; biomarkers; acute toxicity; corresponding relationships

\section{Introduction}

Radix Aconiti (Chuanwu in Chinese), the dried mother root of Aconitum carmichaeli Debx., has been widely used for thousands of years in clinical practice. As a famous and effective traditional Chinese medicine (TCM), it plays an important role in the treatment of diseases, such as rheumatoid joint pain, sciatica, paralysis and others [1]. However, the cardiotoxicity of this Chinese herbal medicine is severe, and the improper use of it will present highly toxicological risk and lead to fatal cardiac poisoning [2]. Aconitine, mesaconitine, hypaconitine and other diester-type alkaloids are known to be the main toxic substances of Radix Aconiti [3]. Modern research also showed that Radix Aconiti has an obvious toxicity caused by aconitine, and it usually produces abnormal heart function, fast respiratory rate, arrhythmia and other symptoms [4]. Generally, biochemical tests and histopathological observation are used to evaluate the safety, but these methods are time-consuming, have low sensitivity, are a heavy workload and have other shortcomings, thus we can not find toxicity during the early stage 
and explain mechanism of toxicity [5]. In the pre-experimental study, we found four acute toxic biomarkers of Radix Aconiti: shikimic acid, L-acetylcarnitine, LysoPC(22:5) and valine. Results showed that these endogenous biomarkers can quickly predict toxicity during the early stages [6]. However, the relationships between endogenous biomarkers and exogenous toxic substances are still not clear. This often hinders research on the toxic mechanism of TCMs. Therefore, there is an urgent need for a fast, accurate approach to find the toxic substances of TCMs, and study the correlations of endogenous biomarkers and exogenous toxic substances.

Metabolomics is a new approach developed following genomics, transcriptomics, and proteomics, and it is an important field of systematic biology [7]. Metabolomics could not only evaluate metabolism of biological samples, identify biomarkers, but also screen the active ingredients or toxic components in TCMs based on the overall efficacy [8]. As a new evaluation method, metabolomic biomarkers have been widely applied for efficacy assessment, toxicity evaluation and disease diagnosis with their unique advantages $[9,10]$. Li et al. has applied ten pre-determined biomarkers to evaluate whether the drugs have potential hepatotoxicity, and the results showed that these biomarkers are rapid, and have strong specificity, and high accuracy [11]. Liquid chromatography-mass spectrometry (LC-MS) is a detection methods that has high sensitivity and good accuracy, is easy operation and has other advantages $[12,13]$. In addition, using LC-MS for biological sample analysis in metabolomics studies, we could quickly and comprehensively obtain information of endogenous metabolites and plasma chemical compositions. Therefore, metabolomics technology and biomarkers were used in this experiment.

Generally, some serum substances of TCMs are the key factors in causing toxicity. The relationships between these exogenous substances and endogenous biomarkers can help explain the toxic mechanism of TCM. Therefore, this experiment was conducted to find relationships between endogenous biomarkers of Radix Aconiti and its exogenous substances. First, we identified toxic substances of Radix Aconiti in rat serum, and analyzed the change trends, followed by which we selected the toxic substances that varied linearly with the dose concentrations, and related to toxicity strength. These selected components were considered as potential toxic substances. Then we found out pre-determined biomarkers of Radix Aconiti by UPLC-Q-TOF-MS technology, and analyzed the variation trends. Finally, Canonical correlation analysis (CCA) was conducted to determine the correspondence between biomarkers and potential toxic substances. Research in this correspondence will help elaborate toxic mechanisms of TCM, and by this newly developed method we can quickly and accurately define the toxic substances in rat serum that really lead to toxic effects.

\section{Results}

\subsection{Animal Behavior}

After intragastric administration with different concentrations of Radix Aconiti ethanol extract, the animals suffered different degrees of abnormal motion. Drowsiness, ataxia, even convulsions, opisthotonos and other symptoms began to appear $30 \mathrm{~min}$ after administration. With the increase of administration concentration, these toxic effects gradually advanced. The toxic effect of high-dose group occurred early, obviously and severely. With the decrease of dosage, occurrence of toxicity grew less. However, the rats in normal saline group remained a normal state, and there was no abnormal behavior.

\subsection{Biochemical Analysis}

The detection and analysis of the enzymatic spectrum of serum are important to evaluate the extent of organ damage caused by toxic effects. In this study, we selected CK and LDH (heart damage detection index), ALT and AST (liver damage monitoring indicators), and Urea and Scr (kidney damage detection index) to evaluate the toxicity of Radix Aconiti. Creatine kinase (CK) and lactate dehydrogenase (LDH) are the commonly used indicators to assess the heart toxicity [14-16]. Their 
levels in the serum from the model group were compared with NS group by t-test. Kidney damage is indicated by increased contents of conventional indicators serum creatinine (Scr) and serum urea in the model groups compared with those in the control group [17,18]. Aspartate aminotransferase (AST), and alanine aminotransferase (ALT) values in the administration and normal control were compared to evaluate liver toxicity [19-21]. The biochemical results of our experiments are described in Figure 1. These biochemical parameters increased in a dose-dependent manner. Compared with control group, CK, AST, Urea and LDH significantly increased in the high dose (10 g/ kg) group of Radix Aconiti extract; LDH also significantly increased in the middle dose group.
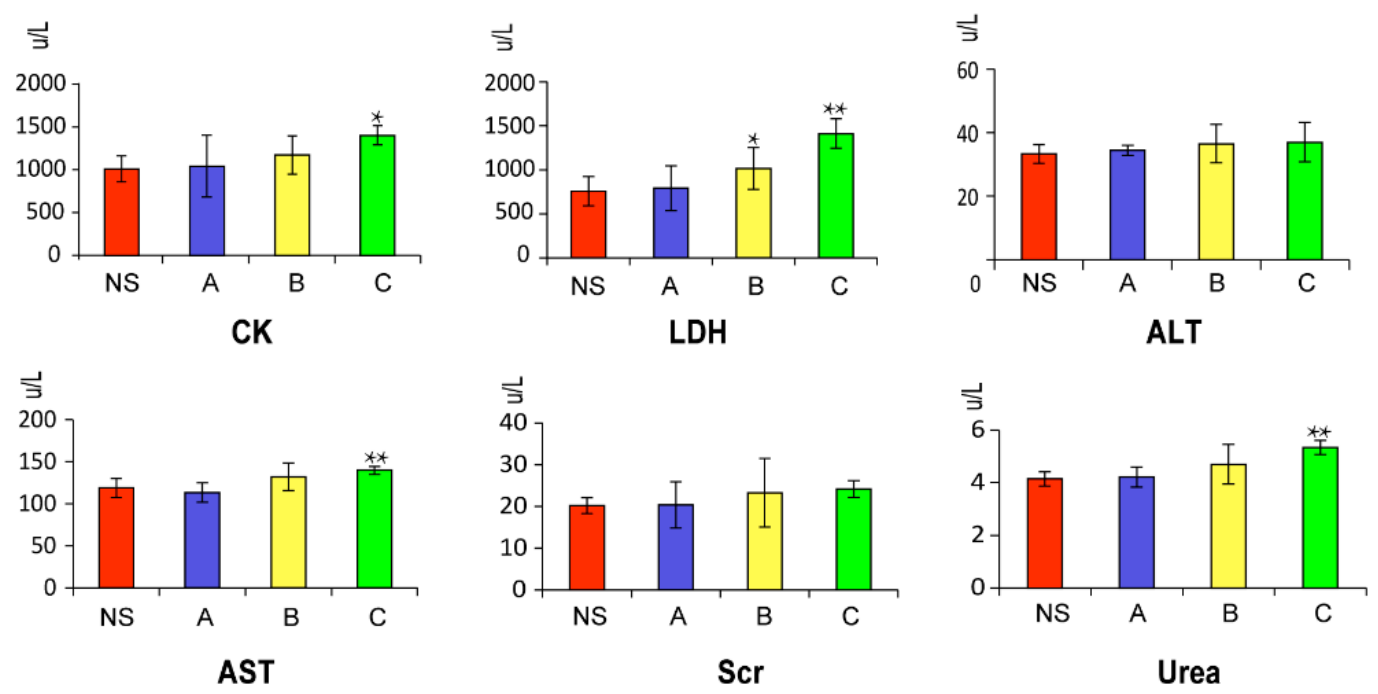

Figure 1. Effect of different concentrations of Radix Aconiti ethanol extract groups on various biochemical parameters in rats. Significant difference from control: ${ }^{*} p<0.05,{ }^{* *} p<0.01$. NS: Normal Saline; A: $2 \mathrm{~g} / \mathrm{kg}$ ethanol extraction; B: $5 \mathrm{~g} / \mathrm{kg}$ ethanol extraction; C: $10 \mathrm{~g} / \mathrm{kg}$ ethanol extraction.

\subsection{Histopathological Assessment}

We used histopathological examination to evaluate the extent of tissue damage. The histopathological results are shown in Figure 2. In the NS group, normal basic structure of rat heart, liver and kidney tissue; arranged regular cardiac muscle fibers; clear structure of hepatic lobules; and normal number, morphology and distribution of glomerular in renal corticomedullary exist. In addition, there are no significant interstitial fibrosis in heart, liver and kidney tissue. In low dose group, part of the heart cell nuclear varies in size, mild edema around the central vein, part distal convoluted tubule and collecting duct atrophy with mild luminal expansion appear. In middle dose group, some dilated blood vessels under the heart tunica, blurred epithelial cavity of proximal tubule, slight edema around the central vein, and small pieces of scattered lymphocytes exist. In high dose group, heart cell nuclear mildly varies in size and within a slight shift, part of the central vein was dilated with slight edema in the surrounding, distal convoluted tubule and the collecting duct scatter epithelium atrophy with small tube expansion occur.

\subsection{Pearson Analysis Results between Exogenous Toxic Substances and Biochemical Indicators for Acute Toxicity of Radix Aconiti}

Typical UPLC-Q-TOF-MS spectrum of the rat serum and plasma fingerprint after intragastrically dosed with Radix Aconiti ethanol extraction is shown in Figures S1 and S2. The QC results of toxic substances and biomarkers met the requirement for testing exogenous and endogenous metabolites (shown in Tables S1 and S2). The basic peak ion (BPI) chromatograms of Radix Aconiti ethanol extraction are shown in Figure S3. According to the fingerprint spectrum, $m / z$ values and $\mathrm{MS}^{2}$ information, we found the exogenous toxic substances which were both contained in rat serum and Radix Aconiti 
ethanol extraction. Finally, eight exogenous toxic substances were identified: karacoline, karacolidine, pengshenine A, demethyl-14-acetylkaracoline, talatisamine, 14-acetyl-talatisamine, mesaconitine and deoxyaconitine. Ion information of these toxic substances are shown in Table 1. The identification process of toxic substances is demonstrated in Supplementary Materials.

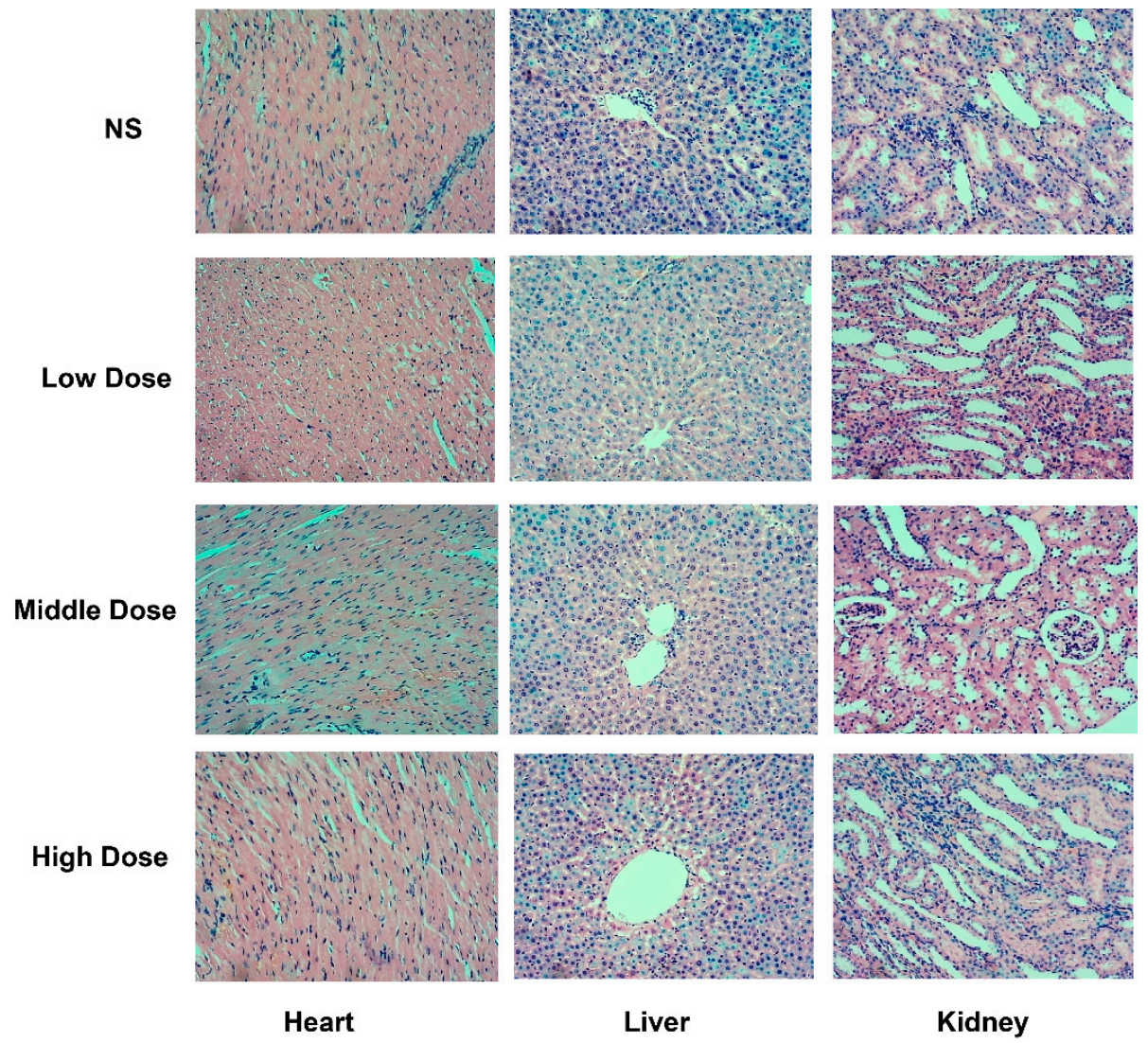

Figure 2. Pathological examination of heart, kidney and liver tissue after the administration of Radix Aconiti ethanol extraction. NS: Normal Saline; Low Dose: $2 \mathrm{~g} / \mathrm{kg}$ ethanol extraction; Middle Dose: $5 \mathrm{~g} / \mathrm{kg}$ ethanol extraction; High Dose: $10 \mathrm{~g} / \mathrm{kg}$ ethanol extraction $(10 \times$ magnification).

Table 1. Mass measurement for exogenous metabolites of Radix Aconiti ethanol extraction.

\begin{tabular}{ccccc}
\hline Metabolites & $\mathbf{T}_{\mathbf{R}}(\mathbf{m i n})$ & $\boldsymbol{m} / \boldsymbol{z}$ & Formula & MS/MS \\
\hline Karacoline & 1.77 & 378.2643 & $\mathrm{C}_{22} \mathrm{H}_{35} \mathrm{NO}_{4}$ & $378.3,360.2,332.2,328.2,310.2$ \\
Karacolidine & 1.82 & 394.2625 & $\mathrm{C}_{22} \mathrm{H}_{35} \mathrm{NO}_{5}$ & $394.3,376.2,358.2,344.2,340.2,326.2$ \\
Demethyl-14-acetylkaracoline & 3.04 & 406.2597 & $\mathrm{C}_{23} \mathrm{H}_{35} \mathrm{NO}_{5}$ & $406.3,388.2,356.2,338.2$ \\
Talatisamine & 6.05 & 422.2967 & $\mathrm{C}_{24} \mathrm{H}_{39} \mathrm{NO}_{5}$ & $422.3,390.3,372.3,358.2,340.2$ \\
14-acetyltalatisamine & 9.4 & 464.3051 & $\mathrm{C}_{26} \mathrm{H}_{41} \mathrm{NO}_{6}$ & $464.3,432.3,400.3,372.2$ \\
Pengshenine A & 1.28 & 436.2327 & $\mathrm{C}_{24} \mathrm{H}_{37} \mathrm{NO}_{6}$ & 436.2 \\
Mesaconitine & 16.82 & 632.3134 & $\mathrm{C}_{33} \mathrm{H}_{45} \mathrm{NO}_{11}$ & $632.3,600.3,582.3,572.3,540.3$ \\
Deoxyaconitine & 18.66 & 630.3338 & $\mathrm{C}_{34} \mathrm{H}_{47} \mathrm{NO}_{10}$ & $630.3,598.3,570.3,538.3,506.3$ \\
\hline
\end{tabular}

We analyzed the variation trends of these toxic substances (shown in Figure 3). As shown in Figure 3, except karacolidine, the other seven toxic substances increased linearly with the increase of concentration. In addition, results of Pearson correlation analysis showed a good correlation between toxic substances and biochemical indicators except pengshenine A (Table 2). As the correlation coefficient approaches 1 , the correlation between two variables is stronger. A positive value indicates a positive correlation, and a negative value indicates a negative correlation. Thus, it was indicated that 
these six toxic substances, which were dose-dependent and related to biochemical indicators, could be accurately considered as acute toxic substances of Radix Aconiti.
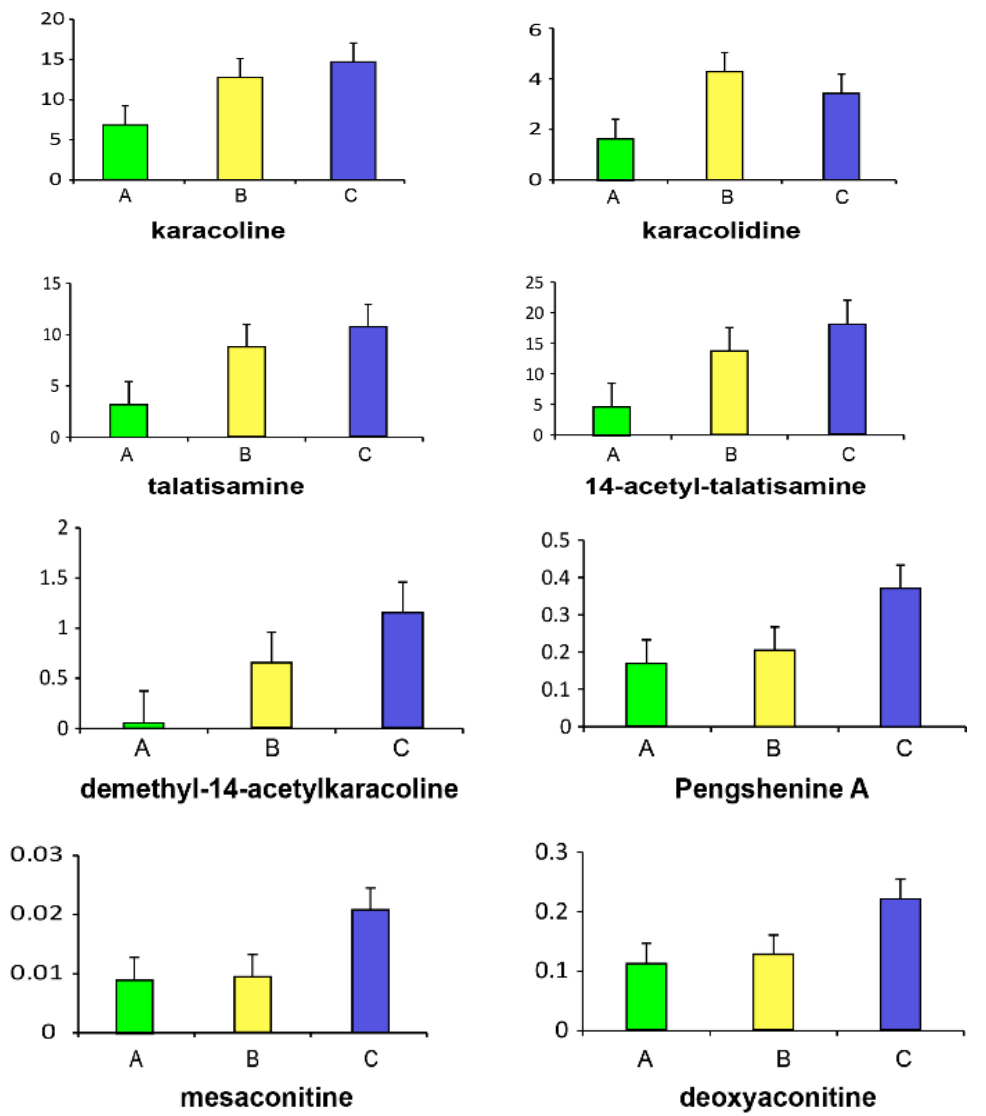

Figure 3. The variation trends of eight serum ingredients' relative contents (peak area intensities) in different concentrations of Radix Aconiti ethanol extraction groups. A: $2 \mathrm{~g} / \mathrm{kg}$ ethanol extraction; B: $5 \mathrm{~g} / \mathrm{kg}$ ethanol extraction; C: $10 \mathrm{~g} / \mathrm{kg}$ ethanol extraction.

Table 2. The correlation of biochemical indicators and exogenous components.

\begin{tabular}{ccccccc}
\hline Metabolites & CK & LDH & ALT & AST & Scr & Urea \\
\hline karacoline & 0.858 & 0.843 & $0.986^{*}$ & 0.810 & 0.916 & 0.888 \\
karacolidine & 0.709 & 0.692 & $0.950^{*}$ & 0.761 & 0.876 & 0.763 \\
demethyl-14-acetylkaracoline & $0.987^{*}$ & 0.985 & 0.942 & $0.966^{*}$ & $0.979 *$ & $0.997^{* *}$ \\
pengshenine A & 0.922 & 0.911 & 0.910 & 0.765 & 0.850 & 0.921 \\
talatisamine & $0.984^{*}$ & $0.978^{*}$ & $0.954^{*}$ & 0.919 & $0.959 *$ & $0.999^{* *}$ \\
14-acetyl-talatisamine & $0.990^{*}$ & $0.985^{*}$ & 0.939 & 0.908 & 0.944 & $0.993 *$ \\
mesaconitine & $0.960^{*}$ & $0.953^{*}$ & 0.888 & 0.805 & 0.861 & $0.951^{*}$ \\
deoxyaconitine & $0.967^{*}$ & $0.958^{*}$ & $0.950^{*}$ & 0.865 & 0.925 & $0.972^{*}$ \\
\hline
\end{tabular}

*: Correlation is significant $(p<0.05)^{* *}$ : Correlation is highly significant $(p<0.01)$.

\subsection{Correspondence between Endogenous Biomarkers and Exogenous Toxic Substances of Radix Aconiti}

Based on the previous study, we found four pre-determined biomarkers, shikimic acid, L-acetylcarnitine, LysoPC(22:5) and valine, and then we drew a histogram of content trends of these biomarkers (Figure 4). Simultaneously, we conducted Pearson correlation analysis between biomarkers and traditional biochemical indicators by SPSS 17 software. Results showed that these biomarkers seemed to be significantly dose-dependent and were obviously relevant to biochemical indicators (Table 3). The identification process of biomarkers is illustrated in Supplementary Material, and ion 
information of shikimic acid, L-acetylcarnitine, LysoPC(22:5) and valine are determined according to our previous study [6].
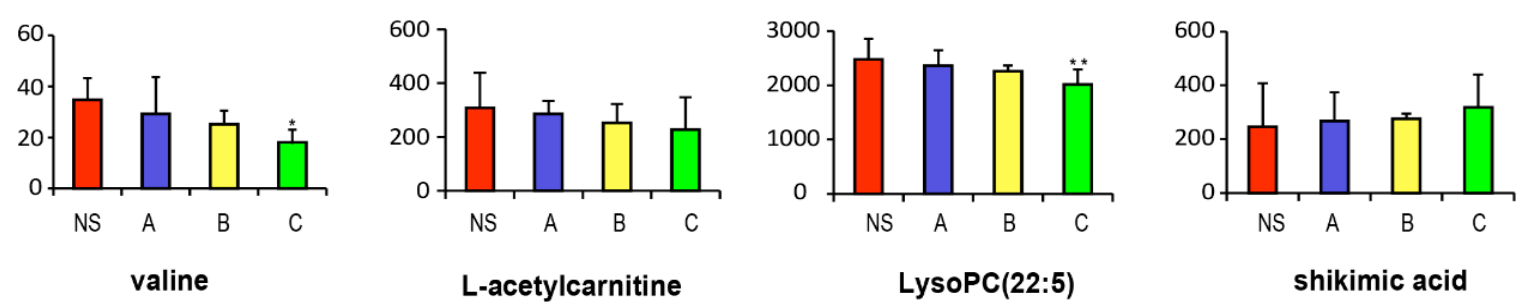

Figure 4. The variation trends of biomarkers' relative contents (peak area intensities) in Radix Aconiti ethanol extraction groups with different doses. Significant difference from control: ${ }^{*} p<0.05$, ** $p<0.01$. NS: Normal Saline; A: $2 \mathrm{~g} / \mathrm{kg}$ ethanol extraction; B: $5 \mathrm{~g} / \mathrm{kg}$ ethanol extraction; C: $10 \mathrm{~g} / \mathrm{kg}$ ethanol extraction.

Table 3. The correlation of biochemical indicators and endogenous biomarkers.

\begin{tabular}{ccccccc}
\hline Metabolites & CK & LDH & ALT & AST & Scr & Urea \\
\hline valine & $-0.963^{*}$ & $-0.954^{*}$ & -0.949 & -0.856 & -0.920 & $-0.968^{*}$ \\
L-acetylcarnitine & $-0.950^{*}$ & $-0.942^{*}$ & $-0.987^{*}$ & -0.907 & $-0.968 *$ & $-0.970^{*}$ \\
LysoPC(22: 5) & $-0.983^{*}$ & $-0.978^{*}$ & -0.915 & -0.866 & -0.908 & $-0.980^{*}$ \\
shikimic acid & $0.974 *$ & $0.969 *$ & 0.881 & 0.828 & 0.870 & $0.964^{*}$ \\
\hline \multicolumn{7}{c}{$*$ Correlation is significant $(p<0.05)}$.
\end{tabular}

$*$ : Correlation is significant $(p<0.05)$.

CCA was made to determine the correspondence between acute toxic dose-dependent biomarkers of Radix Aconiti and its toxic substances. During the CCA, we obtained four pairs of canonical variables, and the canonical correlation coefficients were 1.000, 0.904, 0.792, and 0.470. The closer the value of canonical correlation coefficient is to 1 , the better correlation of endogenous biomarkers and exogenous toxic substances is. Normalization coefficients of biomarkers and toxic substances are shown in Table 4 . Positive and negative coefficients reflect the trend between the indicators, and the absolute value of coefficient reflects the degree of correlation between the indicators. As seen in Table 4, LysoPC(22:5) was highly correlated with 14-acetyl-talatisamine and mesaconitine, and it also had a good correlation with talatisamine and deoxyaconitine. L-acetylcarnitine showed a good negative correlation with demethyl-14-acetylkaracoline and deoxyaconitine. Shikimic acid showed a high positive correlation with karacoline, and it is also related to demethyl-14-acetylkaracoline and deoxyaconitine. Valine was highly positively correlated with deoxyaconitine, and it also had a good negative correlation with talatisamine. All of the results indicated that there really are some relationships between endogenous biomarkers and exogenous toxic substances of Radix Aconiti. However, this needs further research to interpret how they affect each other.

Table 4. The normalization coefficients of acute toxic biomarkers and toxic substances.

\begin{tabular}{ccccc}
\hline \multirow{2}{*}{ Toxic Substances } & \multicolumn{4}{c}{ Biomarkers } \\
\cline { 2 - 5 } & $\mathbf{y 1}$ & $\mathbf{y 2}$ & $\mathbf{y 3}$ & $\mathbf{y 4}$ \\
\hline x1 & 0.403 & -0.191 & 1.270 & 0.136 \\
x2 & -0.287 & -0.636 & 0.658 & -0.229 \\
x3 & -0.587 & -0.223 & 0.224 & -0.558 \\
x4 & -1.111 & 0.097 & 0.427 & -0.309 \\
x5 & 1.444 & -0.258 & -0.353 & -0.138 \\
x6 & 0.585 & -0.555 & -0.832 & 0.867 \\
\hline
\end{tabular}

x1: karacoline; x2: demethyl-14-acetylkaracoline; x3: talatisamine; x4: 14-acetyl-talatisamine; x5: mesaconitine; x6: deoxyaconitine; y1: LysoPC(22: 5); y2: L-acetylcarnitine; y3: shikimic acid; y4: valine. 


\section{Discussion}

3.1. Potential Biological Significance of Exogenous Toxic Substances of Radix Aconiti on the Endogenous Biomarkers

In the results of CCA, LysoPC(22:5) has great correlation with mesaconitine, 14-acetyl-talatisamine, talatisamine and deoxyaconitine. LPCs, also called Lysophospholipids, are a type of endogenous phospholipid. It is a metabolite of PCs phospholipid, and PCs is an important component of biological membranes [6]. In addition, lysophospholipids can transmit lipid signal by means of lysophospholipid receptors (LPL-R). As a G-protein coupled receptor, LPL-R played a portion role of messenger in the organism life activities. [22,23] Some drugs can activate these receptors and send signals to activate lysophospholipids, which can affect the normal function of cardiomyocytes [9]. When cardiotoxicity was induced by traditional Chinese medicine or drug, the protein kinase C (PKC) pathway was activated, and phosphatase A2 activity was enhanced. This may lead to damage of the phospholipid membranes, and LPC reduction [24,25]. The over-activation of PKC may induce myocardial hypertrophy, apoptosis, and lead to structural and functional changes in cardiomyocytes, and, ultimately, affect cardiac function [26].

L-acetylcarnitine showed a good negative correlation with deoxyaconitine and demethyl-14acetylkaracoline. L-acetylcarnitine is an esterified compound of L-carnitine, whose function is similar to L-carnitine. L-carnitine is a key substance of fat metabolism, and it could promote fatty acids into the mitochondria for oxidative decomposition $[27,28]$. Carnitines is thought to be the carrier for transporting fatty acid, and to increase the rate of fatty acids oxidation, reduce the consumption of glycogen [29]. Decrease of carnitines suggested that oxidation of myocardial fatty acid was inhibited, and fatty acid metabolism and sugars aerobic oxidation were reduced. These changes led to energy metabolic imbalance of myocardial cell, and eventually caused arrhythmias and myocardial damage [30].

Radix Aconiti (Chuanwu in Chinese) was already reported as a toxic Chinese herbal medicine because of the presence of diterpene alkaloids [31,32]. In this study, we eventually found six toxic substances: karacoline, demethyl-14-acetylkaracoline, talatisamine, 14-acetyl-talatisamine, mesaconitine and deoxyaconitine. Research has reported that mesaconitine has a greater toxicity on myocardial cells [33]. Weakness of muscules and death from respiratory depression can be induced by karacoline. Talatisamine may lead to brief hypotention and intestinal contractions [34].

\subsection{Significance of Correspondence between Endogenous Biomarkers and Exogenous Toxic Substances of TCM}

Traditional Chinese medicine contains complex chemical compositions. However, only a few individual substances might contribute to efficacy or toxicity. Thus, it is hard to find biologically active ingredients due to many interfering substances. In addition, it is also difficult to investigate pharmacological or toxicological mechanisms caused by various components. Therefore, we proposed a new method in this experiment to find toxic substances of TCM, and study the related mechanism that may occur. Using metabolomic technology and CCA, we found the correlation between endogenous biomarkers and exogenous toxic substances. These relationships had great significance for the research on the confirmation of toxic substances and toxic mechanism of Radix Aconiti. Meanwhile, this method provided an important reference for studying the mechanism of efficacy or toxic effect of TCM. In addition, the overlapping of multi-level analytical methods made the final obtained exogenous toxic substances more accurate. With the same batch of animals, studies of toxic substances and biomarkers for toxic evaluation could be conducted at the same time, which could greatly reduce the cost of research. 


\section{Experimental Section}

\subsection{Reagents and Materials}

The acetonitrile and formic acid of high pressure liquid chromatography (HPLC)-grade were provided by Oceanpak (Gothenburg, Sweden) and ROE SCIENTIFIC INC. (Beijing, China), respectively. Distilled water was purchased from Wahaha (Hangzhou, China). Normal saline (NS) was obtained from Queensland Technology Co., Ltd. (Tianjin, China) [9]. Radix Aconiti was available from AnGuo, HeBei Province (Anguo City, China), and it was authenticated by professor Tianxiang Li (Tianjin University of Traditional Chinese Medicine, Tianjin, China). The contents of mesaconitine, aconitine, and hypaconitine in raw material are $0.2336 \mathrm{mg} / \mathrm{g}, 0.05805 \mathrm{mg} / \mathrm{g}$, and $0.2932 \mathrm{mg} / \mathrm{g}$, respectively (Detailed results of content determination were placed in Supplementary Material).

\subsection{Preparation of Radix Aconiti Extraction}

Radix Aconiti was extracted by ethanol. Crushed herbs (50 g) were extracted with 10 times the amount of $70 \%$ ethanol by refluxed for $60 \mathrm{~min}$ to the residue of which 8 times the amount of $70 \%$ ethanol was added and refluxed for another $60 \mathrm{~min}$. Then, the extracting solution was combined after filtrated, and was concentrated to $1 \mathrm{~g} / \mathrm{mL}$ (amount of crude drug) 70\% ethanol extract sample [6].

\subsection{Experimental Animals and Groups}

Forty male Wistar rats, weighing $(200 \pm 20)$ g, were supplied by the Academy of Military Medical Sciences experimental animal center (Beijing). The animal experiment was performed at the Institute of Radiation Medicine, Chinese Academy of Medical Sciences (Tianjin, China). The rats were housed in an SPF-level lab for one week adaptation, and the growth environment was as follows: $12 \mathrm{~h}$ day and $12 \mathrm{~h}$ night, ambient temperature of $23 \pm 2{ }^{\circ} \mathrm{C}$, ambient humidity of $35 \% \pm 5 \%$. The group, dose, administration mode and sampling time are shown in Table 5.

Table 5. Group, dose, administration mode, and sampling time of rats.

\begin{tabular}{ccccc}
\hline Group & Number & Dose & Administration Mode & Sampling Time \\
\hline NS & 10 & $10 \mathrm{~mL} / \mathrm{kg}$ & Intragastrically, single-dose & $30 \mathrm{~min}$ \\
High dose & 10 & $10 \mathrm{~g} / \mathrm{kg}$ & Intragastrically, single-dose & $30 \mathrm{~min}$ \\
Middle dose & 10 & $5 \mathrm{~g} / \mathrm{kg}$ & Intragastrically, single-dose & $30 \mathrm{~min}$ \\
Low dose & 10 & $2 \mathrm{~g} / \mathrm{kg}$ & Intragastrically, single-dose & $30 \mathrm{~min}$ \\
\hline
\end{tabular}

To minimize the suffering of animals, all experiments were performed in strict accordance with Chinese national laws and local guidelines. The animal study was approved by the Animal Ethics Committee of Tianjin University of Traditional Chinese Medicine with ethical approval number TCM-2012-078F01.

\subsection{Sample Collection and Preparation}

All animals were fasted for $12 \mathrm{~h}$ with access to water before sample collection. Each group was given ethanol extract of Radix Aconiti in corresponding concentration. Then, we took $8 \mathrm{~mL}$ of abdominal aorta blood at $30 \mathrm{~min}$ after dose. One milliliter of blood was placed in normal tubes and centrifuged at $3500 \mathrm{rpm}$ for $10 \mathrm{~min}$ at $4{ }^{\circ} \mathrm{C}$. The obtained supernatant was stored in a $-80{ }^{\circ} \mathrm{C}$ freezer for screening toxic substances. Another $1 \mathrm{~mL}$ of the whole blood was handled and stored in the same conditions, and it was used for conventional biochemical test. Furthermore, we prepared another $1 \mathrm{~mL}$ of blood in heparinized tubes, then handled and stored it in the same method for metabolomics study. The remaining blood samples were placed in a $-80^{\circ} \mathrm{C}$ freezer for storage. After blood collection, we collected the heart, liver and kidney tissues from all rats, and immersed them in $10 \%$ formaldehyde 
solution. Then we observed pathological features of the tissues by haematoxylin and eosin (H\&E) staining $[6,11]$.

\subsection{Chromatographic and Mass Spectrometric Conditions}

In toxic substances study, prior to injection, $3 \mathrm{~mL}$ of acetonitrile was added to $1 \mathrm{~mL}$ of serum to precipitate the proteins, followed by centrifugation at $13,000 \mathrm{rpm}$ for $10 \mathrm{~min}$. The obtained supernatant was concentrated to dryness with nitrogen. Then, the residue was redissolved with $100 \mu \mathrm{L}$ of aqueous methanol (1:1) and centrifuged again to obtain supernatant for analysis. In addition, $20 \mu \mathrm{L}$ of Radix Aconiti ethanol extract was diluted by five times the amount of water, and then it was centrifuged at the same condition to obtain supernatant for analysis. Analysis was performed by a Waters UPLC-Q-TOF-MS system (Waters, Milford, MA, USA). All samples were randomly injected into ACQUITY UPLC HSS $\mathrm{C}_{18}$ column $(2.1 \mathrm{~mm} \times 100 \mathrm{~mm}, 1.7 \mu \mathrm{m}$, Waters $)$ at $40{ }^{\circ} \mathrm{C}$ using $0.1 \%$ formic acid in water (A) and $0.1 \%$ formic acid in acetonitrile (B). The parameters were as follows: the flow rate was $0.3 \mathrm{~mL} / \mathrm{min}$, the injection volume was $5 \mu \mathrm{L}$ and the gradient elution program utilized began with $90 \% \mathrm{~A}$, then $90 \% \mathrm{~A}$ at $0-2 \mathrm{~min}, 90 \%-85 \% \mathrm{~A}$ at $2-7 \mathrm{~min}, 85 \%-70 \% \mathrm{~A}$ at $7-15 \mathrm{~min}, 70 \%-61 \% \mathrm{~A}$ at $15-21 \mathrm{~min}, 61 \%-50 \% \mathrm{~A}$ at $21-25 \mathrm{~min}, 50 \%-1 \% \mathrm{~A}$ at $25-26 \mathrm{~min}, 1 \%-1 \% \mathrm{~A}$ at $26-30 \mathrm{~min}, 1 \%-90 \% \mathrm{~A}$ at 30-30.5 $\mathrm{min}$, and $90 \%-90 \% \mathrm{~A}$ at $30.5-40 \mathrm{~min}$. The eluent was directly introduced to the mass spectrometer. To ensure the stability and repeatability of the systems, plasma samples singled out from each group were pooled as a quality control (QC) sample, which were injected and analyzed at an interval of 10 samples. For mass spectrometry, an electrospray ionization source (ESI) interface was used in positive mode. The MS parameters were fixed as follows: the electrospray capillary voltage was $3.5 \mathrm{kV}$, the drying gas flow was $10 \mathrm{~mL} / \mathrm{min}$, the gas temperature was $325^{\circ} \mathrm{C}$, the fragmentor voltage was $6 \mathrm{kV}$, the desolvation gas flow was $600 \mathrm{~L} / \mathrm{h}$, the nebulizer pressure was $350 \mathrm{psi}$, and the collision energy was 20-30 kV. Data were collected in the range of 50-1000 Da.

Metabolomic analysis was performed under the same conditions with a different gradient profiles. A linear gradient was used as follows: $99 \%-99 \%$ A at $0-0.5 \mathrm{~min}, 99 \%-50 \%$ A at $0.5-2 \mathrm{~min}, 50 \%-1 \% \mathrm{~A}$ at $2-9 \mathrm{~min}, 1 \%-1 \%$ A at $9-10 \mathrm{~min}, 1 \%-99 \%$ A at $10-10.5 \mathrm{~min}$, and $99 \%-99 \%$ A at $10.5-12 \mathrm{~min}$. The eluent was introduced to the mass spectrometer directly. Mass spectrometry analysis was performed on a Waters Q-TOF Premier with the same parameters of metabolomics. After the injection of 10 samples, a pooled sample, the QC sample, followed by a blank was injected in order to ensure the stability and repeatability of the systems $[5,6]$.

\subsection{Data Process}

QC samples were used to validate the precision, reproducibility and stability of the specimen in LC-MS analysis. Twenty chromatographic peaks were randomly selected to calculate the relative standard deviations (RSD) of the peak areas and retention times to evaluate the precision and reproducibility. The RSD of the peak areas and retention times less than $15 \%$ indicated that the sample detection method meets metabolomics requirements. Data of toxic substances were analyzed using Masslynx (Waters) software 4.1. In the exported data, according to $m / z$ values and corresponding MS $^{2}$ information, we identified plasma substances of Radix Aconiti compared with the fingerprint of Radix Aconiti ethanol extraction. Then we analyzed the variation trends of these plasma substances (shown in Figure 3), and determined plasma substances which were dose-dependent. Subsequently, we found the relationships between plasma substances and biochemical indicators by Pearson correlation analysis. Finally, these plasma substances that showed a good correlation with biochemical indicators and had a dependent on dose were considered as toxic substances of Radix Aconiti.

Metabolomics original data were exported by using Masslynx (Waters) software. In the exported data, based on retention time, $\mathrm{m} / \mathrm{z}$ values and corresponding $\mathrm{MS}^{2}$ information, we found pre-determined biomarkers, shikimic acid, L-acetylcarnitine, LysoPC(22:5) and valine, and then we drew a histogram of content trends of these biomarkers (Figure 4). Simultaneously, we conducted Pearson correlation analysis between biomarkers and traditional biochemical indicators by SPSS 
17 software. Finally, we determined the dose-dependent biomarkers that were correlated to biochemical indicators.

\subsection{Canonical Correlation Analysis}

In general, research on the correlation between two sets of variables adopts simple correlation, linear correlation or regression analysis. However, these methods only consider a relationship between the individual evaluation index, and find it difficult to grasp the essence of the problem. CCA is a method of correlating linear relationships between two multidimensional variables. It can more comprehensively reflect the intrinsic relations between the variable groups, and is widely used to study the relationships between the variable groups [35]. In our experiment, exogenous toxic substances were set as $\mathrm{X}$ group and endogenous biomarkers were set as $\mathrm{Y}$ group to conducted CCA by using SPSS 17 software.

\section{Conclusions}

In this study, using biochemical indicators (CK, LDH, ALT, AST, Scr, and Urea) as the bridge, we developed a novel method to find the relationships between endogenous biomarkers and exogenous toxic substances of Radix Aconiti ethanol extraction by UPLC-Q-TOF-MS metabonomic method and CCA. First, we found eight toxic substances of Radix Aconiti and analyzed the correlation between biochemical indicators and these exogenous toxic substances. Then, we found four acute toxic biomarkers of Radix Aconiti based on the previous study, and analyzed the correlation between biochemical indicators and these endogenous biomarkers. Finally, we determined four biomarkers and six toxic substances of Radix Aconiti, which were dose-dependent and related to biochemical indicators. The relationships of these four biomarkers and six toxic substances were analyzed by means of CCA. Results showed that LysoPC(22:5) has different levels of correlation with mesaconitine, talatisamine, 14-acetyl-talatisamine and deoxyaconitine. L-acetylcarnitine showed a good negative correlation with demethyl-14-acetylkaracoline and deoxyaconitine. Shikimic acid was related to karacoline, demethyl-14-acetylkaracoline and deoxyaconitine. Valine was correlated with deoxyaconitine and talatisamine. This method provides a new idea for studying the toxic substances of TCM, and helps us quickly and accurately find toxic substances in rat serum that really lead to toxicity. At the same time, research on the relationships between endogenous biomarkers and exogenous toxic substances of TCM plays a positive role in revealing the complex toxic mechanisms of TCM in vivo.

Supplementary Materials: Supplementary materials can be accessed at: http:/ /www.mdpi.com/1420-3049/21/ $12 / 1623 / \mathrm{s} 1$.

Acknowledgments: This project was supported by the Program for Changjiang Scholars and Innovative Research Team in University (No. IRT 14R41), the National Key Technology R\&D Program (No. 2011BAI07B00, No. 2011BAI07B08), the Natural Science Foundation of Tianjin (No. 15JCYBJC29400), and Science and Technology Development Fund Program for Higher Education Institutions in Tianjin (No. 20140208).

Author Contributions: The authors contributed to this work as follows: Y.L. and Y.X. conceived and designed the study; J.X., Y.W. and B.Y. carried out the experiments; H.Z. and P.Z. analyzed the research data; and H.Z. and Z.H. wrote the manuscript. All authors read and approved the final manuscript.

Conflicts of Interest: The authors declare that they have no competing interests.

\section{References}

1. Zhu, H.; Wang, C.; Qi, Y.; Song, F.; Liu, Z.; Liu, S. Rapid quality assessment of Radix Aconiti Preparata using direct analysis in real time mass spectrometry. Anal. Chim. Acta 2012, 752, 69-77. [CrossRef] [PubMed]

2. Singhuber, J.; Zhu, M.; Prinz, S.; Kopp, B. Aconitum in Traditional Chinese Medicine-A valuable drug or an unpredictable risk? J. Ethnopharmacol. 2009, 126, 18-30. [CrossRef] [PubMed] 
3. Yu, B.; Cao, Y.; Xiong, Y.K. Pharmacokinetics of aconitine-type alkaloids after oral administration of Fuzi (Aconiti Lateralis Radix Praeparata) in rats with chronic heart failure by microdialysis and ultra-high performance liquid chromatography-tandem mass spectrometry. J. Ethnopharmacol. 2015, 165, 173-179. [CrossRef] [PubMed]

4. Wang, W.K.; Hsu, T.L.; Chiang, Y.; Wang, Y.Y. Pulse spectrum study on the effect of sie-zie-tang and Radix Aconiti. Am. J. Chin. Med. 1997, 25, 357-366. [CrossRef] [PubMed]

5. Li, Y.; Deng, H.; Ju, L.; Zhang, X.; Zhang, Z.; Yang, Z.; Wang, L.; Hou, Z.; Zhang, Y. Screening and validation for plasma biomarkers of nephrotoxicity based on metabolomics in male rats. Toxicol. Res. 2016, 5, 259-267. [CrossRef]

6. Li, Y.; Hou, Z.; Wang, Y.; Wang, L.; Ju, L.; Zhang, Z.; Deng, H.; Yuan, L.; Yang, B.; Zhang, Y. Screening and verification of linearly dependent biomarkers with acute toxicity induced by Radix Aconiti based on liquid chromatography-mass spectrometry-based metabolite profiling. RSC Adv. 2015, 5, 103915-103924. [CrossRef]

7. Tan, G.; Lou, Z.; Liao, W.; Zhu, Z.; Dong, X.; Zhang, W.; Li, W.; Chai, Y. Potential biomarkers in mouse myocardium of doxorubicin-induced cardiomyopathy: A metabonomic method and its application. PLOS ONE 2011, 6, e27683. [CrossRef] [PubMed]

8. Qiu, S.; Zhang, A.H.; Sun, H.; Yan, G.L.; Wang, X.J. Overview on metabolomics in traditional Chinese medicine. World J. Pharmacol. 2014, 3, 33-38. [CrossRef]

9. Li, Y.; Ju, L.; Hou, Z.; Deng, H.; Zhang, Z.; Wang, L.; Yang, Z.; Yin, J.; Zhang, Y. Screening, Verification, and Optimization of Biomarkers for Early Prediction of Cardiotoxicity Based on Metabolomics. J. Proteome Res. 2015, 14, 2437-2445. [CrossRef] [PubMed]

10. Cong, W.; Liang, Q.; Li, L.; Shi, J.; Liu, Q.; Feng, Y.; Wang, Y.; Luo, G. Metabonomic study on the cumulative cardiotoxicity of a pirarubicin liposome powder. Talanta 2012, 89, 91-98. [CrossRef] [PubMed]

11. Li, Y.; Wang, L.; Ju, L.; Deng, H.; Zhang, Z.; Hou, Z.; Xie, J.; Wang, Y.; Zhang, Y. A Systematic Strategy for Screening and Application of Specific Biomarkers in Hepatotoxicity Using Metabolomics Combined with ROC Curves and SVMs. Toxicol. Sci. 2016, 150, 390-399. [CrossRef] [PubMed]

12. Xie, G.; Zheng, X.; Qi, X.; Cao, Y.; Chi, Y.; Su, M.; Ni, Y.; Qiu, Y.; Liu, Y.; Li, H.; et al. Metabonomic Evaluation of Melamine-Induced Acute Renal Toxicity in Rats. J. Proteome Res. 2010, 9, 125-133. [CrossRef] [PubMed]

13. Wei, R.; Li, G.; Seymour, A.B. High-Throughput and Multiplexed LC/MS/MRM Method for Targeted Metabolomics. Anal. Chem. 2010, 82, 5527-5533. [CrossRef] [PubMed]

14. Sheela, S.C.; Shyamala, D.C.S. Protective effect of Abana, a poly-herbal formulation, on isoproterenol induced myocardial infarction in rats. Indian J. Pharmacol. 2000, 32, 198-201.

15. Luna, A.; Villanueva, E.; Castellano, M.; Jimenez, G. The determination of CK, LDH and its isoenzymes in pericardial fluid and its application to the post-mortem diagnosis of myocardial infarction. Forensic Sci. Int. 1982, 19, 85-91. [CrossRef]

16. Khalid, M.A.; Ashraf, M. Direct detection of endogenous hydroxyl radical production in cultured adult cardiomyocytes during anoxia and reoxygenation: Is the hydroxyl radical really the most damaging radical species? Circ. Res. 1993, 72, 725-736. [CrossRef] [PubMed]

17. Song, S.; Meyer, M.; Turk, T.R.; Wilde, B.; Feldkamp, T.; Assert, R.; Wu, K.; Kribben, A.; Witzke, O. Serum cystatin $\mathrm{C}$ in mouse models: A reliable and precise marker for renal function and superior to serum creatinine. Nephrol. Dial. Transplant. 2009, 24, 1157-1161. [CrossRef] [PubMed]

18. Yanardag, R.; Bolkent, S.; Ozsoy-Sacan, O.; Karabulut-Bulan, O. The Effects of Chard (Beta vulgaris L. var. cicla) Extract on the Kidney Tissue, Serum Urea and Creatinine Levels of Diabetic Rats. Phytother. Res. 2002, 16, 758-761. [CrossRef] [PubMed]

19. Ahsan, M.R.; Islam, K.M.; Bulbul, I.J.; Musaddik, M.A.; Haque, E. Hepatoprotective Activity of Methanol Extract of Some Medicinal Plants against Carbon Tetrachloride-Induced Hepatotoxicity in Rats. Eur. J. Sci. Res. 2009, 37, 302-310.

20. Ye, X.; Feng, Y.; Tong, Y.; Ng, K.M.; Tsao, S.; Lau, G.K.; Sze, C.; Zhang, Y.; Tang, J.; Shen, J.; et al. Hepatoprotective effects of Coptidis rhizoma aqueous extract on carbon tetrachloride-induced acute liver hepatotoxicity in rats. J. Ethnopharmacol. 2009, 124, 130-136. [CrossRef] [PubMed]

21. Abdualmjid, R.J.; Sergi, C. Hepatotoxic Botanicals-An Evidence-based Systematic Review. J. Pharm. Pharm. Sci. 2013, 16, 376-404. [CrossRef] [PubMed] 
22. Fukushima, N.; Ishii, I.; Contos, J.J.; Weiner, J.A.; Chun, J. Lysophospholipid Receptors. Annu. Rev. Pharmacol. Toxicol. 2001, 41, 507-534. [CrossRef] [PubMed]

23. Xu, Y.; Xiao, Y.J.; Zhu, K.; Baudhuin, L.M.; Lu, J.; Hong, G.; Kim, K.S.; Cristina, K.L.; Song, L.; Williams, F.S.; et al. Unfolding the Pathophysiological Role of Bioactive Lysophospholipids. Curr. Drug Targets Immune Endocr. Metab. Disord. 2003, 3, 23-32. [CrossRef]

24. Fuly, A.L.; Machado, A.L.; Castro, P.; Abrahão, A.; Redner, P.; Lopes, U.G.; Guimarães, J.A.; Koatz, V.L. Lysophosphatidylcholine produced by the phospholipase A2 isolated from Lachesis muta snake venom modulates natural killer activity as a protein kinase C effector. Toxicon 2007, 50, 400-410. [CrossRef] [PubMed]

25. Al Makdessi, S.; Andrieu, J.L.; Herilier, H.; Faucon, G. Effect of isoproterenol on the metabolism of myocardial fatty acids. J. Mol. Cell. Cardiol. 1987, 19, 141-149. [CrossRef]

26. Verma, S.K.; Deshmukh, V.; Liu, P.; Nutter, C.A.; Espejo, R.; Hung, M.L.; Wang, G.S.; Yeo, G.W.; Kuyumcu-Martinez, M.N. Reactivation of fetal splicing programs in diabetic hearts is mediated by protein kinase c signaling. J. Biol. Chem. 2013, 288, 35372-35386. [CrossRef] [PubMed]

27. Zhai, W.; Neuman, S.L.; Latour, M.A.; Hester, P.Y. The Effect of Dietary L-Carnitine on Semen Traits of White Leghorns. Poult. Sci. 2007, 86, 2228-2235. [CrossRef] [PubMed]

28. Kivilompolo, M.; Öhrnberg, L.; Orešič, M.; Hyötyläinen, T. Rapid quantitative analysis of carnitine and acylcarnitines by ultra-high performance -hydrophilic interaction liquid chromatography-tandem mass spectrometry. J. Chromatogr. A 2013, 1292, 189-194. [CrossRef] [PubMed]

29. Liu, Y.T.; Jia, H.M.; Chang, X.; Cheng, W.H.; Zhao, X.; Ding, G.; Zhang, H.W.; Cai, D.Y.; Zou, Z.M. Metabolic pathways involved in Xin-Ke-Shu protecting against myocardial infarction in rats using ultra high-performance liquid chromatography coupled with quadrupole time-of-flight mass spectrometry. J. Pharm. Biomed. Anal. 2014, 90, 35-44. [CrossRef] [PubMed]

30. Arsenian, M.A. Carnitine and its derivatives in cardiovascular disease. Prog. Cardiovasc. Dis. 1997, 40, 265-286. [CrossRef]

31. Wang, J.; Heijden, R.; Spruit, S.; Hankermeier, T.; Chan, K.; van der Greef, J.; Xu, G.; Wang, M. Quality and safety of Chinese herbal medicines guided by a systems biology perspective. J. Ethnopharmacol. 2009, 126, 31-41. [CrossRef] [PubMed]

32. Wang, J.; Heijden, R.; Spijksma, G.; Reijmers, T.; Wang, M.; Xu, G.; Hankemeier, T.; van der Greef, J. Alkaloid profiling of the Chinese herbal medicine Fuzi by combination of matrix-assisted laser desorption ionization mass spectrometry with liquid chromatography-mass spectrometry. J. Chromatogr. A 2009, 1216, 2169-2178. [CrossRef] [PubMed]

33. Zhang, J. Study on the Chemical Constituents and Quality Control of Lateral Roots of Aconitum Carmichaelii Debx; Peking Union Medical College: Beijing, China, 2014.

34. Ulubelen, A.; Kolak, U. Chemical and Biological Studies with an Aconitum and a Delphinium Species. In Innovations in Chemical Biology; Springer: Amsterdam, The Netherlands, 2009; pp. 39-49.

35. Shi, Z.; Liu, Z.; Liu, C.; Wu, M.; Su, H.; Ma, X.; Zang, Y.; Wang, J.; Zhao, Y.; Xiao, X. Spectrum-Effect Relationships Between Chemical Fingerprints and Antibacterial Effects of Lonicerae Japonicae Flos and Lonicerae Flos Base on UPLC and Microcalorimetry. Front. Pharmacol. 2016, 7, 12. [CrossRef] [PubMed]

Sample Availability: Not available.

(C) 2016 by the authors; licensee MDPI, Basel, Switzerland. This article is an open access article distributed under the terms and conditions of the Creative Commons Attribution (CC-BY) license (http://creativecommons.org/licenses/by/4.0/). 